\title{
Chromosomal abnormalities and $Y$ chromosome microdeletions in infertile men from Morocco
}

Yassine Naasse ${ }^{1,3}$, Hicham Charoute ${ }^{1}$, Brahim El Houate ${ }^{1}$, Chadli Elbekkay², Lunda Razoki ${ }^{2}$, Abderrahim Malki ${ }^{3}$, Abdelhamid Barakat ${ }^{1 *}$ and Hassan Rouba ${ }^{1}$

\begin{abstract}
Background: Male infertility is responsible for $50 \%$ of infertile couples. Thirty percent of male infertility is due to cytogenetic and genetic abnormalities. In Arab and North African populations, several studies have shown the association of these chromosomal abnormalities with male infertility. Our objective is to evaluate the frequency of chromosomal abnormalities and $\mathrm{Y}$ chromosome microdeletions in infertile men from Morocco.

Methods: A total of 573 Moroccan infertile men (444 azoospermic and 129 oligozoospermic men) referred for cytogenetic analysis to the Department of Cytogenetics of the Pasteur Institute of Morocco, were screened for the presence of chromosomal abnormalities and $Y$ chromosome microdeletions.

Results: Chromosomal abnormalities accounted for approximately $10.5 \%$ (60/573). Fifty six cases among them have sex chromosome abnormalities (93.34\%), including Klinefelter's syndrome in 41 patients (68.34 \%). Autosomal chromosome abnormalities (6.66 \%) were observed in 4 patients. Chromosomal abnormalities were more prevalent in azoospermic men (13.06 \%) than in oligospermic men (1.55\%). Y microdeletions were detected in 16 of 85 patients (AZFc: $14.12 \%$, AZFbc: $4.70 \%$ ), most of them where azoospermic men with no chromosomal abnormality.

Conclusions: These results highlighted the need for efficient molecular genetic testing in male infertility diagnosis. In addition, a genetic screening should be performed in infertile men before starting assisted reproductive treatments.
\end{abstract}

Keywords: Male infertility, Chromosomal abnormalities, Y microdeletions, Severe oligozoospermia, Azoospermia

\section{Background}

Infertility is a public health problem; approximately $15 \%$ of couples have difficulty or are unable to conceive [1]. Whereas, from $30 \%$ to $50 \%$ of infertility cases are due to a male factor [2]. There are many and diverse causes of male infertility, including accidental causes, congenital birth defects, functional impairments, environmental pollutants, or genetic factors. The latter represents between $10 \%$ and $15 \%$ of severe male infertility $[3,4]$.

Chromosomal anomalies are considered as one of the most important causes of male infertility [5-7]. Their incidence is higher in infertile men than the general population

\footnotetext{
* Correspondence: hamid.barakat@pasteur.ma

'Laboratoire de Génétique Moléculaire Humaine, Département de la Recherche Scientifique, Institut Pasteur du Maroc, 1 Place Louis Pasteur, 20360 Casablanca, Morocco

Full list of author information is available at the end of the article
}

(about 10 times) [8]. Chromosomal abnormalities (structural or numerical) are often detected in azoospermia and severe oligospermia [9], at frequencies ranging from $10 \%$ to $23.62 \%$ and from $1.10 \%$ to $13.33 \%$, respectively [8, 10-14].

Chromosomal abnormalities are not the only genetic causes of male infertility. Deletion of the $\mathrm{Y}$ chromosome region containing the azoospermia factor (AZF) is considered the most common genetic cause of male infertility [15]. Three regions on $\mathrm{Y}$ chromosome long arm (AZFa, AZFb and AZFc) are recurrently deleted in men with severe spermatogenic failure [16]. The frequency of these microdeletions in azoospermic and severe oligospermic men is between $1 \%$ and $50 \%$ [16-18].

This study aimed to assess the different types of $\mathrm{Y}$ chromosome abnormalities and microdeletions, and their frequency in a Moroccan group of infertile men. In addition, we conducted several comparisons between 
frequencies found in the current study and frequencies reported in other populations.

\section{Methods \\ Patients}

A group of 573 Moroccan infertile men were involved in this retrospective study. These patients with azoospermia or severe oligozoospermia were recruited at the Pasteur Institute of Morocco from 1998 to 2013. Patients with azoospermia or severe oligozoospermia resulting from endocrine or obstructive causes were excluded. The average age was $38.70 \pm 7.00$ years. The Karyotype was performed for all patients, while the screening for Y-chromosomal microdeletions was conducted in 85 patients for whom DNA samples were available.

\section{Ethics statement}

We obtained written informed consent from each subject and the study protocol was approved by the local committee on research ethics of the Pasteur Institute of Morocco.

\section{Chromosomal analysis}

Peripheral blood was collected in heparinized vacutainers. The cells were harvested and cultured in the laboratory of cytogenetics. Samples were incubated in an RPMI-1640 solution in the presence of phytohemagglutinin (PHA-E) for $72 \mathrm{~h}$ at $37{ }^{\circ} \mathrm{C}$. $2 \mathrm{~h}$ before the end of the culture, colchicine was added. After centrifugation, the pellet recovered was treated with a hypotonic solution $(0.075 \mathrm{M} \mathrm{KCl})$. The samples were fixed by Carnoy fixative (acetic acid/methanol $1 / 3$ acid). Fixed cell suspensions were spread on glass slides using a Pasteur pipette. These slides were immersed in the fixative Berger. The slides were immersed in the denaturing medium Earle. Then they undergone a Giemsa staining, and finally reading slides by G-banding technique using a microscope connected to a computer through a camera. At least 20 metaphases were counted for each sample.

\section{Y-microdeletions studies}

The genomic DNA extraction was carried out using the Phenol Chloroform standard conventional protocol in the laboratory of Human Molecular Genetics at the Pasteur Institute of Morocco. The samples were lysed by incubation of erythrocytes in Tris-EDTA (TE 20/5) followed by centrifugation. Once the pellet was white, it has undergone lysis of leukocytes by incubation in a solution containing Tris-EDTA (TE 10/5), $20 \%$ SDS and Proteinase $\mathrm{K}$ overnight at $56{ }^{\circ} \mathrm{C}$. Phenol was added and the tube centrifuged. After recovery of the upper aqueous phase, the Chloroform-Isoamyl Alcohol (24/1) was added. After centrifugation, the upper aqueous phase was recovered. Absolute Ethanol was added for DNA precipitation. Once recovered and dried, the DNA was resuspended in Tris-EDTA (TE 10/1) 2 to 3 days and then diluted and stored at $4{ }^{\circ} \mathrm{C}$ before analysis.

Conventional microdeletions in the long arm of the $\mathrm{Y}$ chromosome (Yq) were detected by the polymerase chain reaction (PCR). 6 pairs of oligonucleotide primers were used to amplify the specific Y chromosome STS, which correspond to different loci of the azoospermia factors (AZF). Two sets of multiplex PCR reactions were performed for each patient. The primer pair that amplifies specific STS SRY gene was used as positive control.

The volume of the reaction mixture used in the multiplex PCR was $25 \mu \mathrm{l}$, containing $5 \mu \mathrm{l}$ of DNA $(25 \mathrm{ng} / \mu \mathrm{l})$, $2.5 \mu \mathrm{l}$ of $\mathrm{MgCl}_{2}$ buffer (10X), $2 \mu \mathrm{l}$ of dNTP $(2.5 \mathrm{mM})$ $0.9 \mu \mathrm{l}$ of $\mathrm{MgCl}_{2}(25 \mathrm{mM}), 0.2 \mu \mathrm{l}$ of Taq DNA polymerase (5 units) and $0.8 \mu$ lof each primer $(10 \mathrm{pM})$. On thermocycling, the Touch-Down PCR method was used to increase the specificity and reproducibility of the PCR by avoiding the presence of non-specific bands. The program used an initial denaturation of $5 \mathrm{~min}$ at $94{ }^{\circ} \mathrm{C}$, followed by 5 cycles of $40 \mathrm{~s}$ at $94{ }^{\circ} \mathrm{C}, 45 \mathrm{~s}$ at $60{ }^{\circ} \mathrm{C}$ and $50 \mathrm{~s}$ at $72{ }^{\circ} \mathrm{C}$, followed by 30 cycles of $35 \mathrm{~s}$ at $94{ }^{\circ} \mathrm{C}, 35 \mathrm{~s}$ at $58^{\circ} \mathrm{C}$ and $40 \mathrm{~s}$ at $72{ }^{\circ} \mathrm{C}$, and finally a final elongation of $7 \mathrm{~min}$ at $72{ }^{\circ} \mathrm{C}$. Electrophoresis was performed on $3 \%$ agarose gels containing Ethidium Bromide (EtBr) was performed for the control of the PCR products, using a size marker $100 \mathrm{bp}$. The gel was visualized under ultraviolet light.

\section{Results}

This study was performed on a sample of 573 infertile men, which were separated into two groups: men with non-obstructive azoospermia $(n=444 ; 77.48 \%)$, and men with severe oligospermia $(n=129 ; 22.52 \%)$ (Table 1$)$. The average age is $38.70 \pm 7.00$ years. Cytogenetic analysis showed that among the 573 patients included in the study, 513 men $(89.5 \%)$ had a normal karyotype (46; XY), and 60 men $(10.5 \%)$ had various chromosomal abnormalities. Frequencies of these chromosomal abnormalities in patients with non-obstructive azoospermia and severe oligozoospermia, are $10.12 \%(n=58)$ and $0.35 \%(n=2)$, respectively. Among the 60 patients with chromosomal abnormalities, four ( $0.7 \%)$ had an abnormality of autosomal chromosomes: Inversion 46; XY, inv 9 (p11; p13), and 56 men $(9.8 \%)$ had sex chromosome abnormalities: two mosaic forms 46; XY/47; XXY, one patient with 47; XYY, three patients with 45; X (15\%)/46; XY (85\%), three patients with 46; X, del Yq11, six patients with 46; XX, and the most frequent is Klinefelter syndrome (KFS) 47; XXY, it was found in 41

Table 1 Distribution of different types of infertility

\begin{tabular}{ll}
\hline Type of infertility & $\%$ (No. of men/Total No.) \\
\hline Non obstructive Azoospermia & $77.48(444 / 573)$ \\
Oligozoospermia Severe & $22.52(129 / 573)$ \\
\hline
\end{tabular}


Table 2 Type and frequency of chromosomal anomalies in 573 Moroccan infertile men

\begin{tabular}{llll}
\hline & Non-obstructive Azoospermia & Severe Oligospermia & Overall those with counts $<5$ million/ml \\
\hline Sex Chromosome & & & \\
Aberrations & $8.79 \%(39 / 444)$ & $1.55 \%(2 / 129)$ & $7.16 \%(41 / 573)$ \\
$47 ; X X Y$ & $0.46 \%(2 / 444)$ & $0 \%(0 / 129)$ & $0.35 \%(2 / 573)$ \\
Mosaic $46 ; X Y / 47 ; X X Y$ & $0.23 \%(1 / 444)$ & $0 \%(0 / 129)$ & $0.18 \%(1 / 573)$ \\
$47 ; X Y Y$ & $0.68 \%(3 / 444)$ & $0 \%(0 / 129)$ & $0.53 \%(3 / 573)$ \\
$45 ; X(15 \%) / 46 ; X Y(85 \%)$ & $0.68 \%(3 / 444)$ & $0 \%(0 / 129)$ & $0.53 \%(3 / 573)$ \\
$46 ; X$, del Y q11 & $1.36 \%(6 / 444)$ & $0 \%(0 / 129)$ & $1.05 \%(6 / 573)$ \\
$X X$ males $(46 ; X X)$ & $12.2 \%(54 / 444)$ & $1.55 \%(2 / 129)$ & $9.8 \%(56 / 573)$ \\
Subtotal & & & \\
Autosomal Chromosome & & $0 \%(0 / 129)$ & $0.7 \%(4 / 573)$ \\
Abnormalities & $0.9 \%(4 / 444)$ & $1.55 \%(2 / 129)$ & $10.47 \%(60 / 573)$ \\
Inversion & $13.06 \%(58 / 444)$ & & \\
$46 ; X Y$, inv 9 (p11 ; p13) & & & \\
Total & & & \\
\hline
\end{tabular}

patients (39 azoospermic men, and two men with severe oligozoospermia) (Table 2).

Molecular analysis was performed to detect Y chromosome microdeletions in 85 patients. Among these patients, twelve had a deletion of AZFc (14.12 \%), four cases had a deletion of AZFbc, and 69 cases had an intact $\mathrm{Y}$ chromosome $(81.18 \%)$. No patient with a deletion of AZFa, AZFb or AZFabc was observed (Table 3). The occurrence of AZFc locus deletion is identical among azoospermic and oligospermic (50\%), whereas the AZFbc deletion was found in $75 \%$ of azoospermic and $25 \%$ of oligozoospermic patients (Table 3).

All cases with AZFc or AZFbc locus deletion had a normal karyotype (46; XY). For patients with no deletion $(n=69)$, only one case had the Klinefelter syndrome (47; $\mathrm{XXY}$ ). The remaining patients had a normal karyotype (Table 4).

\section{Discussion}

Numerical and structural chromosome abnormalities played a principal role in male infertility. The prevalence of these abnormalities remained poorly studied and little discussed in the literature, including their prevalence in the Moroccan population.

Studies performed in different populations showed that the incidence of chromosomal abnormalities in infertile men varies between $2.2 \%$ and $19.6 \%$ [19]. In the current study, the frequency of chromosomal abnormalities in infertile men (non-obstructive azoospermic and severe oligozoospermic men) was $10.5 \%$. This frequency is comparable with those reported in Table 5. It is well known that the sperm count is inversely related to the presence of chromosomal abnormalities [20]. The proportion of chromosomal abnormalities in azoospermic men $(13.06 \%)$ was significantly higher than in severe oligospermic men $(1.55 \%)$, these frequencies were consistent with those found in other studies [20].

According to the study conducted by Ferlin et al., Klinefelter's syndrome frequency in infertile men reached respectively $10 \%$ and $5 \%$ in azoospermic and severe oligospermic men [4]. In our patients, we found that the Klinefelter syndrome 47; XXY was the most common in azoospermic men (9.25\%) than in oligospermic severe men (1.55\%). In consistence with other studies [21, 22], the mosaic forms of Klinefelter syndrome was found only in two azoospermic patients. Most men with KS are azoospermic and their ability to conceive remained too low [23]. However, Klinefelter Syndrome patients may have their own children genetically using the technique of testicular sperm extraction (TESE) or micro-TESE followed by ICSI [24]. However, their offspring have an increased risk for chromosomal abnormalities [25].

Structural rearrangements of the Y chromosome, including deletions, ring chromosome and isochromosomes may

Table 3 Type and frequency of Y-microdeletions in 85 infetiles men evaluated

\begin{tabular}{llll}
\hline & Non-obstructive azoospermia & Severe oligospermia & Overall those with counts $<5$ million/ml \\
\hline Absence of Y-microdeletions & $76.81 \%(53 / 69)$ & $23.19 \%(16 / 69)$ & $81.18 \%(69 / 85)$ \\
AZFc microdeletion & $50 \%(6 / 12)$ & $50 \%(6 / 12)$ & $14.12 \%(12 / 85)$ \\
AZFbc microdeletion & $75 \%(3 / 4)$ & $25 \%(1 / 4)$ & $4.70 \%(4 / 85)$ \\
\hline
\end{tabular}


Table 4 Incidence of Y-microdeletions in men with normal karyotype and men with Klinefelter Syndrome

\begin{tabular}{lll}
\hline & $46 ; X Y$ & $47 ; X X Y$ \\
\hline Absence of $Y$ microdeletions & $98.55 \%(68 / 69)$ & $1.45 \%(1 / 69)$ \\
AZFc deletion & $100 \%(12 / 12)$ & $0 \%(0 / 12)$ \\
AZFbc deletion & $100 \%(4 / 4)$ & $0 \%(0 / 4)$ \\
\hline
\end{tabular}

lead to different phenotypes. In this study, we detected three patients with deletion of the Y chromosome long arm, which are all azoospermic men. In fact, the long arm of the $\mathrm{Y}$ chromosome plays a crucial role in the process of spermatogenesis. Partial or total loss of the arm means the loss of genes controlling spermatogenesis, leading to a defect in sperm production [26, 27].

Disorders of sex development (DSD) regroup the clinical situation where the development of the chromosomal, gonadal or anatomical sex is atypical [28]. Three form of the DSD sex caused by chromosome aneuploidy were observed in our sample: The XX male, 47; XYY and $45 ; \mathrm{X} / 46 ; \mathrm{XY}$.

The "XX Male Syndrome" is one of the sex chromosome abnormalities. Men with this syndrome can have phenotype similar to those with Klinefelter's syndrome with normal male external genitalia, but they are sterile [29]. Studies have shown that the frequency of XX males among azoospermic varies between $0.6 \%$ [30] and $3.7 \%$ $[21,22]$. In our study, the frequency of this chromosomal abnormality was $1.36 \%$.

Three patient have the mosaic 45; X (15\%)/46; XY (85\%), and one patient have 47; XYY, all of them are azoospermic. The mosaic form 45; X (15\%)/46; XY (85\%) are described in many studies and the phenotype can range from female phenotype and ambiguous to male phenotype with infertility [30]. The 47; XYY syndrome is associated with behavior difficulties and in some case with infertility. Most males born with these chromosome patterns will go through life without being karyotyped [31]. Until now, no association was found between increased frequency of infertility and this syndrome.

Autosomal abnormalities are less frequent than sex chromosome abnormalities: $0.7 \%$ versus $9.8 \%$. On the other hand, autosomal chromosome abnormalities are detected only in azoospermic men (0.9\%). These results are not similar with those reported in other studies [12, 30, 32]; Amouri et al. reported that gonosomal abnormalities are more common in azoospermic men, while autosomal abnormalities are more common in severe oligospermic men [23].

We identified 4 cases of autosomal chromosome abnormalities $(0.7 \%)$, all of them have the inversion 46 ; $\mathrm{XY}$, inv 9 (p11; p13). This inversion even balanced may be a factor of structural chromosome aberrations during meiosis, and by the way a cause of the disturbances of spermatogenesis.

After the Klinfeleter syndrome, Y chromosome micodeletion are the second most frequent genetic cause of infertility. The frequencies of $\mathrm{Y}$ chromosome microdeletions differ from one study to another, and this may be due to several factors such as the choice of inclusion criteria, the STS markers used, and/or ethnicity of the study population $[33,34]$. In our study, sixteen patients among the 85 analyzed infertile men showed the presence of Y microdeletions (18.83\%). This frequency is in accordance to that found by Ghorbel et al. (17.1\%) [21], Fayez et al. (20.4\%) [22], Imken et al. (3.15\%) [35] and El Oualid et al. (3.83 \%) [36], although our cohort of patients showed a higher frequency, and this may be due to the low number of patients ascertained as well as the

Table 5 Comparison of chromosomal anomalies between this study and other similar studies

\begin{tabular}{|c|c|c|c|c|c|}
\hline \multirow[t]{2}{*}{ Authors } & \multirow[t]{2}{*}{ Regions } & \multirow{2}{*}{$\begin{array}{l}\text { No. } \\
\text { of } \\
\text { cases }\end{array}$} & \multirow{2}{*}{$\begin{array}{l}\text { Frequencies } \\
\%\end{array}$} & \multicolumn{2}{|c|}{ Prevalence of chromosomal aberration \% (No. of men/total No.) } \\
\hline & & & & Non-obstructive Azoospermia & Severe Oligospermia Counts $<5$ million/ml \\
\hline Tuerlings et al., 1998 [45] & Netherlands & 968 & 3.51 & $6.45 \%(4 / 62)$ & $3.47 \%(30 / 865)$ \\
\hline Nagvenkar et al., 2005 [11] & India & 88 & 10.22 & $14.29 \%(6 / 42)$ & $6.52 \%(3 / 46)$ \\
\hline Mohammed et al., 2007 [33] & Kuwait & 289 & 7.95 & $19.44 \%(21 / 108)$ & $1.10 \%(2 / 181)$ \\
\hline Ng et al., 2009 [12] & Hong Kong & 295 & 5.08 & $21.10 \%(5 / 71)$ & $4.46 \%(10 / 224)$ \\
\hline Kosar et al., 2010 [5] & South of Turkey & 115 & 4.34 & $5.43 \%(5 / 92)$ & $0 \%(0 / 23)$ \\
\hline Alkhalaf et al., 2010 [46] & Kuwait & 142 & 18.30 & data not available & data not available \\
\hline Mafra et al., 2011 [34] & Brazil & 143 & 6.29 & $11.62 \%(5 / 43)$ & $4 \%(4 / 100)$ \\
\hline Zhang et al., 2012 [32] & Northeast China & 135 & 14.07 & $17.28 \%(14 / 81)$ & $9.26 \%(5 / 54)$ \\
\hline Cavkaytar et al., 2012 [47] & Turkey & 332 & 7.23 & $11.22 \%(22 / 196)$ & $1.47 \%(2 / 136)$ \\
\hline Amouri et al., 2014 [30] & Tunisia & 476 & 10.92 & $14.10 \%(46 / 328)$ & $4.05 \%(6 / 148)$ \\
\hline Our study & Morocco & 573 & 10.47 & $13.06 \%(58 / 444)$ & $1.55 \%(2 / 129)$ \\
\hline
\end{tabular}

No.: number 
high number of patients with $\mathrm{Y}$ microdeletions, but higher than that found in the Chinese population (6.4\%; $8.5 \%$; $9.1 \%)[12,37,38]$ or other Arab populations (Kuwait (2.6\%) [33], Tunisia (2.7 \% and $6.85 \%$ ) [39, 40], Saudi Arabia (3.2 \%) [41] and Egypt (4\%) [42]. However, the frequency found in this study was included in the range of frequencies of wide world (1-55\%) [17, 18]. These microdeletions are more common in azoospermic $(n=9)$ than severe oligospermic $(n=7)$, and this proportion was found in many reports [33, 35, 37-40, 42].

In this report, we identified only deletions of the AZFc and AZFbc region, while no case with deletion of AZFa and/or AZFb was identified. This result is similar to that described in $\mathrm{Ng}$ et al. [12] and Imken et al. [35] reports, but different to the results found in other reports $[17,34,42,43]$. Several studies have shown that AZFc deletion was the most common [22, 39, 40], and these results are in accordance with ours (14.12\%). However, other studies showed conflicting results [33, 35, 42, 44].

In this study, we showed that sixteen cases with microdeletions (AZFc and AZFbc) have no chromosomal abnormalities (normal karyotype 46; XY) (Table 4). While Ng et al. showed that all patients with AZFc deletion had a normal karyotype 46; XY, while all patients with AZFbc deletion had chromosomal abnormalities [12].

\section{Conclusion}

The prevalence of chromosomal abnormalities and Y chromosome microdeletions are comparable to that found in different parts of the world (North Africa, Asia and Europe). Indeed, the analysis of these genetic factors (karyotype, Y microdeletions, etc. ...) is highly recommended, to identify the causes of infertility and to choose the appropriate assisted reproduction technique, and also to reduce the risk of transmission of these genetic defects to the future generations.

\section{Availability of data and materials}

Not applicable.

\begin{abstract}
Abbreviations
AZF: Azoospermia Factor; dNTP: Triphosphate Deoxyribonucleotides; DSD: Disorders of Sex Development; EDTA: Ethylenediaminetetraacetic acid; BET: Ethidium Bromide; ICSI: Intracytoplasmic Sperm Injection; KCl: Potassium Chloride; KFS: Klinefelter Syndrome; PCR: Polymerase Chain Reaction; PHAE: Phytohemagglutinin- Erythrocytes; RPMI: Roswell Park Memorial Institute medium; SDS: Sodium Dodecyl Sulfate; SRY: Sex-determining Region of Y chromosome; STS: Sequence-tagged site; TE: Tris-EDTA; TESE: Testicular Sperm Extraction.
\end{abstract}

\section{Competing interests}

The authors declare that they have no competing interests.

\section{Authors' contributions}

$A B$ and $H R$ conceived, designed and coordinated the study. YN performed the laboratory work. BE helped in the laboratory work. YN and $\mathrm{HC}$ carried out statistical analysis. YN wrote the paper. CE, LR and AM participated in interpretation of results. All authors read and approved the final manuscript.
Authors' information

Not applicable.

\section{Acknowledgments}

We thank all patients for their cooperation. This work was supported by Institut Pasteur du Maroc. The authors would also like to thank Dr. Kenneth McElreavey for his contribution to this work.

\section{Funding}

This work received no specific grant from any funding agency.

\section{Author details}

${ }^{1}$ Laboratoire de Génétique Moléculaire Humaine, Département de la Recherche Scientifique, Institut Pasteur du Maroc, 1 Place Louis Pasteur, 20360 Casablanca, Morocco. '2Laboratoire de Cytogénétique, Département de la Recherche Scientifique, Institut Pasteur du Maroc, 1 Place Louis Pasteur, 20360 Casablanca, Morocco. ${ }^{3}$ Laboratoire de Physiopathologie et Génétique Moléculaire, Faculté des Sciences Ben M'Sik, Université Hassan II, Casablanca, Morocco.

Received: 30 April 2015 Accepted: 10 September 2015

Published online: 18 September 2015

\section{References}

1. Van Assche E, Bonduelle M, Tournaye H, Joris H, Verheyen G, Devroey P, et al. Cytogenetics of infertile men. Hum Reprod. 1996:4:1-24.

2. Gurunath S, Pandian Z, Anderson RA, Bhattacharya S. Defining infertility - a systematic review of prevalence studies. Hum Reprod Update. 2011;17:575-88.

3. Kim ED, Lipshultz LI. Male subfertility: diagnostic and therapeutic advances. $\mathrm{Br} J$ Urol. 1997:80(4):633-41.

4. Ferlin A, Arredi B, Foresta C. Genetic causes of male infertility. Reprod Toxicol. 2006;22:133-41.

5. Kosar PA, Ozcelik N, Kosar A. Cytogenetic abnormalities detected in patients with non-obstructive azoospermia and severe oligozoospermia. J Assist Reprod Genet. 2010;27:17-21.

6. Dohle GR, Halley DJ, Van Hemel JO, van den Ouwel AM, Pieters MH, Weber $\mathrm{RF}$, et al. Genetic risk factors in infertile men with severe oligozoospermia and azoospermia. Hum Reprod. 2002;17:13-6.

7. Martin RH. Cytogenetic determinants of male fertility. Hum Reprod Updat. 2008;14:379-90.

8. Ceylan GG, Ceylan C, Elyas H. Genetic anomalies in patients with severe oligozoospermia and azoospermia in eastern Turkey: a prospective study. Genet Mol Res. 2009;8:915-22.

9. Chandley A. Chromosome anomalies and $Y$ chromosome microdeletions as casual factors in male infertility. Hum Reprod. 1998;13:45-50.

10. Balkan M, Tekes S, Gedik A. Cytogenetic and Y chromosome microdeletion screening studies in infertile males with oligozoospermia and azoospermia in Southeast Turkey. J Assist Reprod Genet. 2008;25:559-65.

11. Nagvenkar P, Desai K, Hinduja I, Zaveri K. Chromosomal studies in infertile men with oligozoospermia \& non-obstructive azoospermia. Indian J Med Res. 2005;122:34-42.

12. Ng PP, Tang MH, Lau ET, Ng LK, Ng EH, Tam PC, et al. Chromosomal anomalies and Y-microdeletions among Chinese subfertile men in Hong Kong. Hong Kong Med J. 2009;15:31-8.

13. Chiang HS, Wei HJ, Chen YT. Genetic screening for patients with azoospermia and severe oligo-asthenospermia. Int J Androl. 2000;23:20-5.

14. Zhou-Cun A, Yang Y, Zhang SZ, Zhang W, Lin L. Chromosomal abnormality and $Y$ chromosome microdeletion in Chinese patients with azoospermia or severe oligozoospermia. Yi Chuan Xue Bao. 2006;33:111-16.

15. Krausz C, Degl'Innocenti S. Y chromosome and male infertility: update. Front Biosci. 2006;11:3049-61.

16. Vogt PH, Edelmann A, Kirsch S, Henegariu O, Hirschmann P, Kiesewetter F, et al. Human $Y$ chromosome azoospermia factors (AZF) mapped to different subregions in Yq11.1. Hum Mol Genet. 1996;5:933-44.

17. Foresta C, Ferlin A, Garolla A, Moro E, Pistorello M, Barbaux S, et al. High frequency of well-defined Y-chromosome deletions in idiopathic Sertoli cell-only syndrome. Hum Reprod. 1998;13(2):302-07.

18. van der Ven K, Montag M, Peschka B, Leygraaf J, Schwanitz G, Haidl G, et al. Combined cytogenetic and $Y$ chromosome microdeletion screening in males undergoing intracytoplasmic sperm injection. Mol Hum Reprod. 1997;3:699-704. 
19. Nakamura Y, Kitamura M, Nishimura K, Koga M, Kondoh N, Takeyama M, et al. Chromosomal variants among 1790 infertile men. Int J Urol. 2001;8:49-52.

20. Lissitsina J, Mikelsaar R, Punab M. Cytogenetic analyses in infertile men. Arch Androl. 2006;52:91-5.

21. Ghorbel M, Gargouri Baklouti S, Ben Abdallah F, Zribi N, Cherif M, Keskes R, et al. Chromosomal defects in infertile men with poor semen quality. J Assist Reprod Genet. 2012;29:451-56.

22. Fayez AG, El-Sayed AS, El-Desouky MA, Zarouk WA, Kamel AK, Fahmi IM, et al. Molecular Characterization of Some Genetic Factors Controlling Spermatogenesis in Egyptian Patients with Male Infertility. Int J Infertility Fetal Med. 2012;3:69-77.

23. Smyth CM, Bremner WJ. Klinefelter syndrome. Arch Intern Med. 1998;158:1309-14.

24. Krausz C. Male infertility: pathogenesis and clinical diagnosis. Best Pract Res Clin Endocrinol Metab. 2011;25:271-85.

25. Ron-El R, Strassburger D, Gelman-Kohan S, Friedler S, Raziel A, Appelman Z. A 47, XXY fetus conceived after ICSI of spermatozoa from a patient with non-mosaic Klinefelter's syndrome: case report. Hum Reprod. 2000;15(8):1804-06.

26. Valetto A, Bertini V, Rapalini E, Baldinotti F, Di Martino D, Simi P. Molecular and cytogenetic characterization of a structural rearrangement of the $Y$ chromosome in an azoospermic man. Fertil Steril. 2004;81:1388-90.

27. Siffroi JP, Chantot-Bastaraud S, Ravel C. Genetic origin of spermatogenesis impairments: clinical aspects and relationships with mouse models of infertility. Gynecol Obstet Fertil. 2003;31:504-15.

28. Lee PA, Houk CP, Ahmed SF, Hughes IA. Consensus statement on management of intersex disorders. International Consensus Conference on Intersex. Pediatrics. 2006;1 18:488-500.

29. Boucekkine C, Toublanc JE, Abbas N, Chaabouni S, Ouahid S, Semrouni M, et al. Clinical and anatomical spectrum in XX sex reversed patients. Relationship to the presence of $Y$ specific DNA-sequences. Clin Endocrinol (Oxford). 1994;40:733-42.

30. Amouri A, Hammami W, Kilani O, Bouzouita A, Ayed W, Ben Meftah M, et al. Chromosomal evaluation in a group of Tunisian patients with nonobstructive azoospermia and severe oligozoospermia attending a Tunisian cytogenetic department. C R Biologies. 2014;337:223-28.

31. Abramsky L, Chapple J. 47, XXY (Klinefelter syndrome) and 47, XYY: estimated rates of and indication for postnatal diagnosis with implications for prenatal counseling. Prenat Diagn. 1997;17:363-68.

32. Zhang ZB, Jiang YT, Yun X, Yang X, Wang RX, Dai RL, et al. Male infertility in Northeast China: a cytogenetic study of 135 patients with non-obstructive azoospermia and severe oligozoospermia. J Assist Reprod Genet. 2012:29:83-7.

33. Mohammed F, Al-Yatama F, Al-Bader M, Tayel SM, Gouda S, Naguib KK. Primary male infertility in Kuwait: a cytogenetic and molecular study of 289 infertile Kuwaiti patients. Andrologia. 2007;39:87-92.

34. Mafra FA, Christofolini DM, Bianco B, Gava MM, Glina S, Belangero SI, et al. Chromosomal and molecular abnormalities in a group of Brazilian infertile men with severe oligozoospermia or non-obstructive azoospermia attending an infertility service. Int Braz J Urol. 2011;37:244-50.

35. Imken L, El Houate B, Chafik A, Nahili H, Boulouiz R, Abidi O, et al. AZF microdeletions and partial deletions of AZFc region on the $Y$ chromosome in Moroccan men. Asian J Androl. 2007;9:674-78.

36. Eloualid A, Rhaissi H, Reguig A, Bounaceur S, El Houate B, Abidi O, et al. Association of spermatogenic failure with the b2/b3 partial AZFc deletion. PLoS One. 2012;7:e34902.

37. Tse JY, Yeung WS, Ng EH, Zhu HB, Teng XM, Liu YK, et al. A comparative study of $Y$ chromosome microdeletions in infertile males from two Chinese populations. J Assist Reprod Genet. 2002;19:376-83.

38. Tse JY, Yeung WS, Lau EY, Ng EH, So WW, Ho PC. Deletions within the azoospermia factor subregions of the $Y$ chromosome in Hong Kong Chinese men with severe malefactor infertility: controlled clinical study. Hong Kong Med J. 2000;6:143-46.

39. Hammami W, Kilani O, Ben Khelifa M, Ayed W, Abdelhak S, Bouzouita A, et al. Prevalence of $Y$ chromosome microdeletions in infertile Tunisian men. Ann Biol Clin (Paris). 2014;72:331-6.

40. Rejeb I, M'rad R, Maazoul F, Trabelsi M, Ben Jemaa L, Chaabouni M, et al. Y chromosome microdeletions in Tunisian infertile males. PatBio. 2008:56:111-5.

41. Hellani A, Al-Hassan S, labal M, Coskun S. Y chromosome microdeletions in infertile men with idiopathic oligo or azoospermia. J Exp Clin Assist Reprod. 2006:3:1-6.
42. Nowier SR, El-sheikh MM, Rasool HAA, Ismail S. Prevalence of $Y$ Chromosome Microdeletion in Males with Azospermia And Severe Oligospermia in Egypt. Res J Med Medical Sci. 2009;4:189.

43. Simoni M, Bakker E, Krausz C. EAA/EMQN best practice guidelines for molecular diagnosis of Y-chromosomal microdeletions. State of art 2004. Int J Androl. 2004:27:240-49.

44. Elhawary NA, Seif-Eldin NS, Zaki M, Diab H. Azoospermia factor microdeletions: common tag STSs in infertile men with azoospermia and sever oligospermia from Egypt. BMC Genomics. 2014;15:25.

45. Tuerlings J, de France HF, Hamers A, Hordijk R, Van Hemel JO, Hansson K, et al. Chromosome studies in 1792 males prior to intracytoplasmic sperm injection: the Dutch experience. Eur J Hum Genet. 1998;6:194-200.

46. Alkhalaf M, Al-Shoumer K. Cytogenetic abnormalities and azoospermia factor (AZF) microdeletions in infertile men from Kuwait. J Mol Genet Med. 2010:26:232-34.

47. Cavkaytar S, Batioglu S, Gunel M, Ceylaner S, Karaer A. Genetic evaluation of severe male factor infertility in Turkey: a cross-sectional study. Hum Fertil. 2012;15:100-06.9.

\section{Submit your next manuscript to BioMed Central and take full advantage of:}

- Convenient online submission

- Thorough peer review

- No space constraints or color figure charges

- Immediate publication on acceptance

- Inclusion in PubMed, CAS, Scopus and Google Scholar

- Research which is freely available for redistribution 